\title{
MEMORIA PENINSULAR DE UN LEGADO PARA \\ EL SIGLO XXI: FRANCISCO SUÁREZ (1617-2017)
}

\author{
MANUEL LÁZARO PULIDO \\ UNED / U. Bernardo O'Higgins* \\ $M^{\mathrm{a}}$ IDOYA ZORROZA HUARTE \\ Universidad Pontificia de Salamanca
}

\begin{abstract}
RESUMEN: El trabajo presenta las distintas actividades científicas (congresos, jornadas internacionales, publicaciones) que con motivo del cuarto centenario del fallecimiento de Francisco Suárez se han celebrado y se han organizando durante el año 2017 o el curso académico 2017-18. En las iniciativas presentadas se advierte la vigencia de la reflexión suareciana, no sólo por su relevancia histórica, sino también como un pensamiento con el que poder replantear los retos de nuestra situación contemporánea.
\end{abstract}

PALABRAS CLAVE: Suárez; Congresos; publicaciones; Filosofía; Pensamiento barroco.

\section{Peninsular memory of a legacy to XXI Century: Francisco Suárez (1617-2017)}

ABSTRACT: This paper introduce the different scientific activities (congresses, international conferences and meetings, publications) that have been prepared and that are being organized between 2017 and 2018, in occasion of the fourth centenary of the death of Francisco Suarez. These activities show the intellectual wealth of the Suarezian thought, not only because of its historical relevance, but also as a thought for rethinking our contemporary time.

KEY WORDS: Suárez; Congresses; publications; Philosophy; Baroque Philosophy and Thought.

\section{Centenario de un Pensamiento para un nuevo Siglo}

Durante el año 2017 se han celebrado en la Península Ibérica (España y Portugal) diversas actividades para recordar la figura, obra y pensamiento del jesuita español Francisco Suárez. Actividades que se irán cerrando a lo largo del año académico 2017-2018. La figura intelectual de Francisco Suárez es, sin duda, una de las más importantes del pensamiento de la llamada «segunda escolástica», un ejemplo de la profundidad filosófica realizada en la Península Ibérica, y símbolo de la profundidad conceptual de una filosofía y una teología especulativa y práctica. Su original pensamiento dentro de la profundización de la tradición filosófico-teológica que se refleja en la propia metodología de las Disputaciones metafísicas, provoca que haya sido entendido, bien como el último representante de la tradición escolástica en relación a la relectura humanista de la escolástica medieval, bien como el primer eslabón de un

Trabajo dentro del proyecto de investigación financiado por el Departamento de Ciencias del Derecho de la Universidad Bernardo O'Higgins en consonancia con el proyecto «Pensamento Filosófico - Teológico na Península Ibérica (Idade Média e Modernidade)», Unviersidade Católica Portuguesa / CEFi - Centro de Estudos de Filosofia. 
pensamiento ya moderno, bien como el eje necesario de la mentalidad premoderna y barroca.

La riqueza y amplitud de su perspectiva, que es origen de su difícil $-\mathrm{o}$ de su amplia- caracterización, también ha provocado que, aunque no haya sido olvidado, haya sido malinterpretado, o generado reacciones muy diversas. No obstante, poco a poco, se ha ido imponiendo desde la inevitable diversidad hermenéutica y de intereses (metafísicos, analíticos, jurídicos, políticos o teológicos) la realidad de su potencialidad especulativa, tanto considerada intrínsecamente, como en lo que ha supuesto para la historia del pensamiento, siendo revitalizado en sí mismo y desde el propio suarismo ${ }^{1}$ que se ha hecho presente también en el siglo $\mathrm{XX}^{2}$.

Para el mes de septiembre (los días 19-21) de 2017, en la Facultad de Filosofía y Letras de la Universidad Nacional Autónoma de México, se preparó un acto académico, un Coloquio, en recuerdo de la figura del maestro granadino bajo el título: "El siglo de oro español y Francisco Suárez. Su legado tras 400 años de ausencia» ${ }^{3}$. El título del Coloquio, al que nos referiremos más tarde, tenía una intención que podría leerse de forma ambivalente, en la medida en que la "ausencia» podría interpretarse como 400 años sin la presencia, por su muerte, de Suárez, o, la «ausencia» como silenciamiento de la figura de Suárez en el panorama filosófico, teológico y del pensamiento jurídico-político. Si es cierto que el panorama filosófico contemporáneo ha cambiado, como hemos señalado, también lo es que ahora las tesis de Francisco Suárez resisten el envite de una contemporaneidad dominada por la postmetafísica, la filosofía analítica, el posthumanismo y la fenomenología, entre otras, no sólo porque su propuesta invita a dialogar con las diversas posiciones presentes en el debate filosófico actual, sino porque su obra da la posibilidad de poder desplegar un sólido pensamiento con capacidad de revitalizarse allí donde las nuevas perspectivas hacen agua o dejan un regusto intelectual de vacuidad. También porque comprender la filosofía suareciana nos lleva a comprender mejor la problemática surgida en la modernidad, convirtiéndose asimismo en un elemento clave para la realización de una coherente historia de la filosofía.

Esta actualidad de Francisco Suárez en el panorama filosófico-teológico es una realidad corroborada por los datos. La celebración del centenario de su nacimiento, allá por 1948, ya supuso en su tiempo un aldabonazo a un

\footnotetext{
1 Cf. Gómez Caffarena, J., "Suarismo», en Sacramentum Mundi, Barcelona, Herder, 1976, vol. 6, pp. 468-473.

2 Cf. Elorduy, E., «El suarismo en el ambiente postconciliar», en de Alcorta y Echevarria, J. I. (ed.), Philosophia. Miscelánea, Barcelona, Bosch, 1971, pp. 209-218.

3 El encuentro fue fruto de un proyecto de investigación PAPIIT (IN404016): Crisis de la escolástica, humanismo del siglo de oros español y su influencia en México, que se puso en marcha con la organización de otro encuentro en el año 2014 titulado Libros que terminan en la hoguera. 400 años de la condena de la obra política de Francisco Suárez, Facultad de Filosofía y Letras de la Universidad Nacional Autónoma de México, 10-11 de noviembre de 2014. En el encuentro del 2017 participaron 35 investigadores de España, México o Chile en ponencias plenarias y 9 mesas temáticas.
} 
pensamiento que siempre ha estado presente, como evidenciaba el número publicado para la ocasión por la revista Pensamiento ${ }^{4}$. En su Bibliografía suareciana - publicada en Cuadernos de Pensamiento Español-, Jean-Paul Coujou señalaba algunos elencos bibliográficos que ponían en evidencia la vivencia y vigencia de Francisco Suárez. Su propio libro, publicado en 2010, mostraba 157 páginas de referencias bibliográficas ${ }^{5}$ en las que el autor señalaba bibliografías previas como la de Ernest-Marie Rivière (1917-1918) ${ }^{6}$, la de Ceferino Santos-Escudero $(1980)^{7}$, la de Laszlo Polgar (1990) y la de Jacob Schmutz (www.schoslasticon.fr) ${ }^{9}$ que está acompañada de un amplio elenco de «instrumentos bibliográficos» ${ }^{10}$. Coujou justificaba esta obra como reflejo, a su vez, de "la vitalidad de la investigación sobre esta temática» ${ }^{11}$, vitalidad que se ha evidenciado con más claridad si cabe en este año, en el que la recuperación y constante relectura de la filosofía y la teología especulativa y práctica de Suárez se nos ofrece al intelectual de hoy como un pensamiento fecundo para un nuevo siglo.

Como señalaba en una entrevista realizada con motivo de uno de los eventos científicos realizados este 2017 (las Reuniones Filosóficas celebradas en la Universidad de Navarra), José Ángel García Cuadrado sintetizaba la genialidad y actualidad del Eximio con estas palabras:

Suárez es ciertamente un pensador genial, pero también un hombre de su tiempo. Las ideas que él expone de manera profunda, clara y sistemática son el reflejo de toda una época que se apoya en categorías filosóficas de la escolástica medieval y su renovación por parte de la escolástica ibérica desde el siglo XV. Con ellas tiene que dar cuenta de los profundos cambios que llegan con la Modernidad. Una Modernidad que implica un giro hacia la subjetividad, principalmente desde el punto de vista especulativo, pero también en el ámbito de la vida social y política, donde se comienza a tomar conciencia de los derechos individuales. Además, las nuevas ideas científicas comienzan a modificar la visión del mundo. Los problemas filosóficos abordados por Suárez, una vez despojados de su ropaje escolástico, son tremendamente actuales: piénsese, por

4 «Suárez en el IV Centenario de su nacimiento», en Pensamiento, 4 (1948), número extraordinario, $646 \mathrm{pp}$. Existieron otros números especiales y conmemorativos. Al respecto cf. Coujou, J.-P., «Estudios sobre la obra de Suárez», en Bibliografía suareciana, Pamplona, Servicio de Publicaciones de la Universidad de Navarra, 2010, pp. 37-38.

5 Ibid., pp. 9-168.

6 Rivière, P. F. y Scorraille, R. de, Suárez et son oeuvre. I. La Bibliographie des ouvrages imprimés et inédites. II. La doctrine, Toulouse - Barcelona, 1917; cf. Razón y fe, 47 (1917), pp. 442 ss.

Santos-Escudero C., "Bibliografía suareciana de 1948 a 1980», en Cuadernos Salmantinos de Filosofía, 7 (1980), pp. 337-375.

8 Polgar, L., Bibliographie sur l'histoire de la compagnie de Jésus (1901-1980), Roma, Institutum historicum S.I., 1990, pp. 268-329.

9 Scholasticon, página web creada por Jacob Schmutz permanentemente actualizada, disponible en www.schoslasticon.fr, especialmente en http://scholasticon.ish-lyon.cnrs.fr/ Database/Scholastiques_fr.php?ID=1212

10 Cousou, J.-P., Bibliografía suareciana, pp. 29-35.

$11 \quad$ Ibid., p. 7. 
ejemplo, en su tratado sobre la guerra justa. En definitiva, comprender mejor a Suárez es comenzar a comprender mejor los orígenes de nuestros propios modos de pensar ${ }^{12}$.

\section{LOS CONGRESOS Y ENCUENTROS CIENTÍFICOS}

La convocatoria de Suárez ha sido un acicate de revitalización del pensamiento barroco y peninsular en diálogo con la tradición anterior y en su proyección filosófica a la modernidad e incluso el pensamiento contemporáneo. En este sentido, los diversos encuentros que han tenido lugar —o que se están organizando en fechas próximas - han confirmado una red de instituciones académicas y de investigación que son una muestra de la vitalidad de los estudios de la escolástica peninsular, del Renacimiento y Barroco peninsulares, de un lado, y, en el caso que nos atañe, de Suárez, de otro.

\subsection{Madrid. Jornada: "El alcance del pensamiento de Francisco Suárez»}

El 29 de noviembre de 2016, en la Facultad de Filosofía de la Universidad Eclesiástica San Dámaso y organizada por este centro universitario madrileño, se celebró una Jornada titulada El alcance del pensamiento de Francisco Suárez. Este acto que tuvo lugar a finales de 2016, significó una primera mirada en el aniversario del cuarto centenario de la muerte del Doctor Eximio.

Aunque desarrollada la jornada en un día, sin duda alguna constituyó un muy "sabroso aperitivo» académico, a la altura del acontecimiento que se estaba por celebrar a lo largo de 2017.

Las ponencias fueron amplias en tiempo y con discusión, con una duración total de una hora. La primera ponencia estuvo a cargo del profesor Juan Belda (Universidad de Navarra) que disertó sobre Francisco Suárez, heredero de una generación de sabios. Siguieron por la mañana las ponencias de Costantino Esposito (Università degli Studi de Bari Aldo Moro): Suárez, Kant e il problema dell'ontologia moderna; Jean François Courtine (Université Paris IV-Sorbonne): Anthropologie et théologie politique suarézienne.

Por la tarde tuvieron lugar dos ponencias más, las de Harald Schöndorf SJ (Hochschule für Philosophie, Munich): Algunos comentarios sobre Dios como creador en el pensamiento de Suárez; y Nicolás Álvarez de las Asturias (Universidad Eclesiástica San Dámaso): Suárez en el caminar histórico del derecho canónico: El caso de la interpretación de la ley.

Concluyó el encuentro con una comunicación de media hora titulada Suárez y la modernidad europea: filosofía política y doctrina impositiva, de los profesores

12 Palabras del profesor J. A. García Cuadrado recogidas por Nagore Gil en: «La Universidad acogerá las LIII Reuniones Filosóficas entre el 26 y 28 de abril», 2n Vida Universitaria. Disponible en http://www.unav.edu/web/vida-universitaria/detallenoticiapestania3/2017/03/ 27/liii-reuniones-filosoficas:- $\%$ E2\%80\%9Cfrancisco-suarez:-escolastica-y-mundo-moderno\% E2\%80\%9D?articleId=13250875. Consultado el 19 de septiembre de 2017. 
León $M^{\mathrm{a}}$ Gómez Rivas (Universidad Complutense de Madrid) y Francisco Tadeo Baciero Ruiz (Universidad de Salamanca) y con media hora para preguntas y diálogo con los ponentes.

\subsection{Salamanca. IV Encuentro Internacional de Historia del Pensamiento: «Entre el Renacimiento y la Modernidad: Francisco Suárez (1548-1617)»}

Los días 30 y 31 de marzo de 2017, se celebró en la Facultad de Filosofía de la Universidad de Salamanca (día 30) y en la Universidad Pontificia de Salamanca (día 31), el IV Encuentro Internacional de Historia del Pensamiento: "Entre el Renacimiento y la Modernidad: Francisco Suárez (1548-1617)». Como indica su nombre se trataba de la última edición de los encuentros que en torno a la Escuela de Salamanca y el pensamiento escolástico y barroco peninsular ${ }^{13}$ se están celebrando en Salamanca (y que es símbolo presente de esta tradición filosófica y de los estudios históricos de una época tan importante para la historia del pensamiento). El Congreso se realizó animado por los coordinadores José Luis Fuertes Herreros (Universidad de Salamanca), $\mathbf{M}^{\mathrm{a}}$ Idoya Zorroza Huarte (Universidad de Navarra, actualmente en la Universidad Pontificia de Salamanca), Manuel Lázaro Pulido (CEDEU - Universidad Rey Juan Carlos, Instituto Teológico de Cáceres - UPSA, y Universidade Católica Portuguesa, actualmente en la Universidad Nacional de Educación a Distancia) y Ángel Poncela González (Universidad de Salamanca), y con la ayuda inestimable de la Facultad de Filosofía de la Universidad Pontificia de Salamanca representados por su decana Rosa María Herrera García.

A la llamada del pensamiento sobre Suárez acudieron las siguientes instituciones: el Departamento de Filosofía, Lógica y Estética. Facultad de Filosofía. Universidad de Salamanca (España); la Universidade Católica Portuguesa. Faculdade de Teología - Porto / CEFi - Centro de Estudos de Filosofia, Faculdade de Ciências Humanas (Portugal); la Línea Especial de Pensamiento clásico español, Departamento de Filosofía de la Universidad de Navarra (España); el Instituto Teológico de Cáceres, Centro afiliado a la Facultad de Teología de la Universidad Pontificia de Salamanca (España); el CEDEU - Centro de Estudios Universitarios, Centro adscrito a la Universidad Rey Juan Carlos (España); el Departamento de Ciencias del Derecho de la Universidad Bernardo O'Higgins, Santiago (Chile); el Departamento de Filosofía y Humanidades de la Universidad Pontificia Comillas (España); la Faculté de Lettres de la Université de Fribourg (Suiza); el DISUM - Departamento di

13 Las tres ediciones anteriores coordinadas por José Luis Fuertes Herreros, Manuel Lázaro Pulido y $\mathrm{M}^{\mathrm{a}}$ Idoya Zorroza Huarte cubrieron los siguientes temas: «Las pasiones y las virtudes en la época de El Greco» (I Encuentro, 2014); «El pensamiento místico y la Escuela de Salamanca. V Centenario del nacimiento de Teresa de Ávila (1515-2015)» (II Encuentro, 2015); «Las pasiones en el Barroco: de la acedia escolástica a la melancolía moderna» (III Encuentro, 2016). 
Studi Humanistici de la Università degli Studi di Bari «Aldo Moro» (Italia); y el Institut de Estudis Hispànics en la Modernitat, Universitat de las Illes Balears (España). De entre las numerosas intervenciones, ponencias de veinte minutos, que comprimieron el programa (que pese a lo apretado de su desarrollo, no dejó fuera un rico diálogo entre los asistentes, en el que se reflejaba el entusiasmo de los participantes), podemos destacar las siguientes: Jean-Paul Coujou (Institute Catholique de Toulouse/Institut Michel Villey): El estatuto ontológico y político del individuo en Suárez. La persistencia de la huella de la herencia de Ockham en la modernidad.

- Leopoldo José Prieto López (Universidad Francisco de Vitoria): An lex sit aliquid voluntatis: la antropología subyacente a la noción suareciana de ley.

- Simona Langella (Università degli studi di Genova): El tiranicidio: de Francisco de Vitoria a Francisco Suárez.

- Cintia Faraco (Università degli Studi de Napoli «Federico II»): La reflexión en la libertad como fundamento de la dignidad humana: el tirano y el derecho de resistencia.

- Martín González Fernández (Universidad de Santiago de Compostela): Guerra y paz: de Erasmo a Suárez.

- Emanuele Lacca (Universidad de Bohemia del Sur de České Budějovice): ¿Bellum non est instrinsece malum? Guerra y justicia en el De charitate de Francisco Suárez.

- Rafael Ramis Barceló (Universitat de las Illes Balears - IEHM): La configuración y el desarrollo universitario del suarismo en el siglo XVII.

- Samuel Dimas (Universidade Católica Portuguesa / CEFi): A relação entre providência divina e liberdade humana no debate da Escolástica Ibérica sobre a Redenção e a Glória.

- Giancarlo Colacicco (Scuola Normale Superiore di Pisa): Antes de la publicación de las Disputationes metaphysicae (1597). Francisco Suárez y Cristóbal de los Cobos sobre las causas y los principios'.

- Ángel Poncela (Universidad de Salamanca): Suárez y el nominalismo. Una relectura de la sexta Disputación Metafísica.

- Ceferino P.D. Muñoz (CONICET-UNCuyo-UMendoza): Antecedentes de la ontología en la escolástica: de Suárez a Cayetano (Video conferencia).

- Costantino Esposito (Università degli Studi de Bari Aldo Moro): Francisco Suarez e il rischio dell'onto-teo-logia.

- Ana Andaluz Romanillos (Universidad Pontificia de Salamanca): Presencia de Suárez en la filosofía moderna: Kant, Metafísica - Dohna.

- Mauro Mantovani (Università Pontificia Salesiana): La demostrabilidad de la existencia de Dios: Francisco Suárez en el contexto de los maestros jesuitas de Teología y en la lectura de Pedro de Godoy, OP.

- Idoya Zorroza. (Universidad de Navarra): La noción de facultad en Suárez. Entre la antropología y el derecho.

- Olivier Ribordy (Université de Fribourg): Les discussions de Suárez et Patrizi sur l'univers: différents modèles cosmologiques à l'aube de la modernité. 
- Luis Carlos Amezúa Amezúa (Universidad de Valladolid): El dominium altum en Francisco Suárez: un esbozo.

- Francisco Tadeo Baciero Ruiz (Universidad de Salamanca): La polémica Hobbes-Bramhall sobre la noción suareciana de libertad.

- José Ángel García Cuadrado (Universidad de Navarra): El arbitraje internacional en la obra de Francisco Suárez: A propósito de una antigua polémica.

- Daniel Heider (University of South Bohemia): Was Suárez a Perceptual Representationalist?

- Ilaria Acquaviva. (Scuola Alti Studi Collegio San Carlo di Modena): La realitas objectalis in Francisco Suárez e la sua influenza sulla Schulmetaphysik.

- Ignacio Verdú Berganza (Universidad Pontificia Comillas): Francisco Suárez y René Descartes. Una reflexión acerca de la modernidad.

- Valentín Fernández Polanco (Universidad Complutense de Madrid): La importancia de la obra suareciana en la configuración del pensamiento político moderno.

- Francisco Javier Cortés Sánchez (Universidad de Salamanca): Individuo y comunidad en Francisco Suárez. Perspectiva de Eduardo Nicol.

- José Luis Fuertes Herreros (Universidad de Salamanca): Significado de la filosofía de Suárez.

\subsection{Pamplona. LIII Reuniones Filosóficas: «Francisco Suárez: Escolástica y Mundo Moderno»}

Los días 26-28 de abril de 2017 se celebraron las muy consolidadas Reuniones Filosóficas en su LIII edición. El Departamento de Filosofía de la Universidad de Navarra tuvo el acierto de consagrarlo al pensamiento y obra de Francisco Suárez recalcando la proyección de su especulación escolástica en la modernidad con el título clarividente de «Francisco Suárez: Escolástica y Mundo Moderno». En el Comité Organizador, continuando la labor de dirección que había iniciado Ángel Luis González, fallecido un año antes (y a quien se realizó un sentido homenaje el último día de dichas Reuniones), se encontraban Agustín Echavarría (Director), José Ángel García Cuadrado (Subdirector), y María Idoya Zorroza (Secretaria). En el Comité Científico Víctor Sanz Santacruz, Rubén Pereda, Cruz González Ayesta, Enrique Moros y Juan Fernando Sellés. Estas Reuniones suponían el cierre de una serie de workshops y simposios en los que se preparaba el centenario estudiando la obra suareciana desde enfoques complementarios: Perspectivas del 'De legibus' de Francisco Suárez (2014); El tratado De anima de Francisco Suárez. y su proyección en la filosofía moderna (2015) y La proyección de F. Suárez a la filosofía moderna (2016).

Nos sirve de introducción y estado de la cuestión de este Congreso, la presentación del mismo: 
A lo largo del siglo XX la obra de Francisco Suárez (1548-1617) ha despertado un interés creciente entre los historiadores de la filosofía. En la actualidad reina un consenso generalizado en considerarlo la figura clave para comprender el paso del mundo medieval al moderno. Sin embargo, la valoración de su pensamiento sigue siendo muy dispar. Para algunos es el culmen de la escolástica con todas sus virtualidades y defectos; para otros es el principal responsable de la crisis de una metafísica realista. Mientras que para sus detractores Suárez es el eslabón fundamental que explica la deriva idealista, subjetivista del voluntarismo moderno, para sus defensores el Doctor Eximio es un pensador audaz y libre de prejuicios que se cuestionó el ingenuo objetivismo medieval, y elaboró una filosofía de la libertad a la altura de los tiempos.

En todo caso, la amplitud y profundidad de las cuestiones abordadas en su obra resultan encomiables: desde las controversias sobre la gracia, hasta el tratamiento exhaustivo de la metafísica, pasando por el derecho internacional o la guerra justa. Bastaría citar sus Disputationes Metaphysicae o el De legibus para advertir la profunda huella que ha dejado su pensamiento. Estas Reuniones Filosóficas —celebradas en el marco del IV Centenario de su muerte-aspiran a arrojar un poco más de luz, con una crítica cabal, de la obra de esta figura intelectual de primer orden ${ }^{14}$.

El formato adoptado fue el de Ponencias plenarias y 10 Mesas temáticas de ponencias sobre: «Suárez y la filosofía moderna», "Suárez y la teoría del conocimiento», "Suárez y la metafísica», "Suárez y la filosofía del derecho», «Suárez jesuita», "La antropología de Suárez», "Suárez, la metafísica y la teología», "Suárez, antropología y derecho», "La filosofía política de Suárez». Hacemos memoria de las ponencias principales:

- José Ángel García Cuadrado (Universidad de Navarra, Pamplona): Suárez, entre el absolutismo y la democracia

- Salvador Castellote (Facultad de Teología San Vicente Ferrer, Valencia): La 'visio Dei' en Suárez

- Alejandro Vigo (Universidad de Navarra, Pamplona): Verdad trascendental

- Daniel Heider (University of South Bohemia): Suárez, on the Functional Scope of Imaginative Power

- Agustín Echavarría (Universidad de Navarra, Pamplona): La 'ratio futuritionis' y la doble reducción de la 'ciencia media' según Suárez.

- Robert Matava (Christendom College, Alexandria, VA, EE.UU.): Francisco Suárez on the Ontological Status of Divine Action: Implications for the Freewill Debate

- Víctor Salas (Sacred Heart Major Seminary, Detroit, EE.UU): The Christological Structure of Francisco Suárez's Metaphysics

- Robert Fastiggi (Sacred Heart Major Seminary, Detroit, EE.UU): Ser Independiente y Ser Dependiente: una clave para la Metafísica de Suárez

14 Cf. LIII Reuniones Filosóficas: «Francisco Suárez: Escolástica y Mundo Moderno». Disponible en http://www.unav.edu/web/facultad-de-filosofia-y-letras/eventos/liii-reunionesfilosoficas/presentacion. Consultado el 24 de septiembre de 2017. 
- Jean-Paul Coujou (Institute Catholique de Toulouse/Institut Michel Villey): Formes platoniciennes et universel suarézien

\subsection{Porto-Coimbra. $I^{\mathrm{o}}$ Encontro Internacional Pensar o Barroco em Portugal: "Os fundamentos antropológicos e teóricos do pensamento jurídico e político em F. Suárez e na Segunda Escolástica: contexto e projecções. IV Centenário da Morte de Francisco Suárez (1617-2017)».}

A finales del mes de junio (26-28) de 2017 se celebró el $I^{\circ}$ Encontro Internacional Pensar o Barroco em Portugal: "Os fundamentos antropológicos e teóricos do pensamento jurídico e político em F. Suárez e na Segunda Escolástica: contexto e projecções IV $V^{\circ}$ Centenário da Morte de Francisco Suárez (1617-2017)». Organizados por los profesores Manuel Lázaro Pulido (CEDEU - Universidad Rey Juan Carlos, Instituto Teológico de Cáceres - UPSA, y Universidade Católica Portuguesa, actualmente en la Universidad Nacional de Educación a Distancia) y Mário Santiago de Carvalho (Universidade de Coimbra). En esta ocasión el pensamiento de Suárez ha sido el acontecimiento que ha dado lugar a lo que se espera sea una serie de encuentros en Portugal donde se piense la filosofía de la época barroca.

El Encuentro tuvo lugar en el campus de Foz de la Universidade Católica Portuguesa, Centro Regional de Porto (días 26 y 28), y en la Universidade de Coimbra (día 27). Ambientados en el ámbito occidental de la Península Ibérica, y una vez que ya se había celebrado en la ciudad de Salamanca un encuentro en el que muchos de los presentes ya habían participado, parecía interesante visitar la Universidad de Coimbra siguiendo así los pasos vitales e intelectuales de Francisco Suárez, después de haber compartido los espacios de la Ciudad del Tormes. En cierta forma los Congresos de Salamanca y de Porto-Coimbra se diseñaron en una cierta continuidad de formación de una comunidad y una red de pensamiento filosófico a nivel peninsular y europeo.

El Encuentro estuvo organizado por el Centro de Estudos de Filosofia CEFi, Universidade Católica Portuguesa, C. R. Porto (Portugal); el Instituto de Estudos Filosóficos da Faculdade de Letras da Universidade de Coimbra (Portugal); y el Instituto Teológico «San Pedro de Alcántara» de Cáceres. Centro Afiliado a la Universidad Pontificia de Salamanca (España). Colaboraron el Departamento de Filosofia, Comunicação e Informação da Faculdade de Letras da Universidade de Coimbra, Biblioteca Geral da Universidade de Coimbra (Portugal); el Departamento de Teologia Sistemática da Faculdade de Teologia. Universida de Católica Portuguesa, C. R. Porto (Portugal); el Departamento de Filosofía, Lógica y Estética de la Universidad de Salamanca (España); la Línea Especial Pensamiento Clásico Español. Universidad de Navarra (España); el Instituto Teológico de Cáceres, Centro afiliado. Universidad Pontificia de Salamanca (España); CEDEU - Centro de Estudos Universitarios. Centro adscrito. Universidad Rey Juan Carlos (España); la Faculté de Lettes, Université de Fribourg (Suza); el DISUM - Dipartimento di Studi Umanistici. Università degli Studi di Bari «Aldo Moro»(Italia); el Departamento de 
Filosofía e antropoloxia. Universidade de Santiago de Compostela (España); el Institut d'Estudis Hispànics en la Modernitat. Universitat de las Illes Balears (España); y el Departamento de Ciencias del Derecho de la Universidad Bernardo O'Higgins (Chile), contando con el apoyo científico u de gestión de la Ed. Sindéresis, SOFIME (Sociedad de Filosofía Medieval) y del Instituto de Humanidades Francesco Petrarca y de la Fundação para Ciência e a Tecnologia.

Como sucedió en el Congreso de Salamanca, todas las intervenciones fueron ponencias de veinte minutos. Podríamos englobarlas en dos grupos temáticos, uno más cercano al pensamiento práctico (jurídico-político) y otro más especulativo (que incluían propuestas de metafísica, antropología o teología).

\section{A. Pensamiento jurídico-político:}

- Jean-Paul Coujou (Institute Catholique de Toulouse/Institut Michel Villey): Paix et pouvoir chez Marsile de Padoue et Suárez. Les enjeux d'un débat furtif et pourtant décisif sur le théologico-politique.

- Pedro Calafate (Universidade de Lisboa): O princípio da igualdade natural entre as soberanias do orbe como fundamento da comunidade internacionale garantia da paz no pensamento de Francisco Suárez e na segunda escolástica.

- Cintia Faraco (Universidad de Naples «Federico II»): Reflexión sobre la obligación politíca y la libertad en el pensamiento de Francisco Suárez.

- Pablo Font Oporto (Universidad Loyola Andalucía): La articulación operativa del poder político en Suárez: sentido y límites de su entrega al rey por parte del pueblo.

- Juan Carlos Utrera García (Universidad Nacional de Educación a Distancia): Voluntad divina, poder y ley en Francisco Suárez. Notas sobre la teología política de la modernidad.

- Francisco Tadeo Baciero Ruiz (Universidad de Salamanca): Los tratados jurídico-políticos de Locke previos a los Dos Tratados sobre el gobierno civil y su relación con Suárez.

- Amalia Xochitl López Molina (Universidad Nacional Autónoma de México): La libertad humana y el derecho de gentes.

- Roberto H. Pich (Pontíficia Universidade de Rio Grande do Sul): On the Footsteps of Suarez Probabilistic Thought in Morality and Law: Diego de Avendaño S.J. (1594-1688) on Good Conscience, Legal Obligation, and Moral Freedom.

- Simona Langella (Università degli studi di Genova): La guerra justa: peripecias y dislocaciones de un concepto de Vitoria a Suárez.

- Daniel Schwartz (Hebrew University of Jerusalem): La guerra como duelo colectivo en Suárez.

- Ángel Poncela (Universidad de Salamanca): Compromisos metafísicos y epistemológicos del voluntarismo jurídico-político Suareciano.

- Juan Antonio Gómez García (Universidad Nacional de Educación a Distancia): El voluntarismo en la concepción suareciana de ley: bases para una interpretación analógica. 
- Mauricio Lecón Rosales (Universidad Panamericana): ¿Puede una mujer gobernar? Francisco Suárez y la legtimidad de una reina.

- Miguel Régio de Almeida (Universidade de Coimbra): Nótulas para uma revisão crítica do pensamento suareziano na Filosofia dos Direitos Humanos.

- André Santos Campos (Universidade Nova de Lisboa): Francisco Suárez e as tradições do contrato social.

- Leonor Durão Barroso (Universidade Católica Portuguesa): A refutação do absolutismo no pensamento político de Francisco Suárez.

- Emanuele Lacca (University of South Bohemia): Utrum ignorantia excuset poenam legis. Un caso de aplicación de la ley en el De Legibus de Francisco Suárez.

- António Manuel Martins (Universidade de Coimbra): Suárez e o direito de resistência.

- Gonzalo Letelier Widow (Universidad de los Andes): Suárez y el «estado de naturaleza».

- M ${ }^{\mathrm{a}}$ Idoya Zorroza Huarte (Universidad de Navarra): Dos miradas a la vinculación de uso, propiedad y libertad: Suárez y Locke.

- Manuel Lázaro Pulido (UCP-CEFi; CEDEU-URJC; U. Bernardo O’Higgins): El carácter enmendativo y reeducativo de la ley penal en Francisco Suárez.

B. Filosofía especulativa (metafísica, antropología y teología)

- Martín González Fernández (Universidade de Santiago de Compostela): Francisco Suárez y el automatismo animal.

- Simone Guidi (Instituto de Estudos Filosóficos, Universidade de Coimbra): Quantity Matters. The Reception of Suárez's Theory of Extension Before Descartes.

- Giancarlo Colacicco (Scuola Normale Superiore, Pisa): Dios, el ser y la ratio principii: Francisco Suárez, comentador barroco de Tomás de Aquino.

- Constance Blackwell (Foundation for Intellectual History): Cicero and Fonseca's attack on Socrates.

- Daniel Heider (University of South Bohemia): Suárez on Self-Awareness: Embodied Souls, Separated Souls and Angels.

- Rafael Ramón Guerrero (Universidad Complutense de Madrid): Precedente medieval de la metafísica como Tratado: de Avicena y a Suárez.

- Vicente Llamas Roig (Instituto Teológico de Murcia, Pontificia Università Antonianum): La analogia entis en la metafísica suarista. Notas para un patrón mixto.

- Laura Alicia Soto Rangel (Universidad Nacional Autónoma de México): El problema de la libertad en las Disputaciones Metafísicas. Anotaciones sobre la causalidad eficiente.

- Maria da Conceição Camps (Instituto de Estudos Filosóficos - Universidade de Coimbra): O concurso da Graça na perfeição da natureza humana, segundo Francisco Suárez.

- Gilles Olivo (Université de Caen): La verdad en Suarez. 
- Vincent Carraud (Université Paris-Sorbonne): Métaphysique et science des causes.

- Olivier Ribordy (Université de Fribourg): Suárez et Descartes: quelques différences sur l'indifférence. Réflexions croisées sur les fondements anthropologiques de la liberté.

- Rui Lobo (Universidade de Coimbra, FCTUC): The Jesuit buildings of Coimbra - An architectural history.

- Maia do Amaral (Universidade de Coimbra - BGUC): Em Torno de Suárez. Acervo Bibliográfico em Coimbra.

- Costantino Esposito. (Università degli Studi de Bari Aldo Moro): Natura e sovranatura nel pensiero barocco di Francisco Suarez

- Francisco León Florido (Universidad Complutense de Madrid ): La modernidad de Francisco Suárez en la interpretación de André de Muralt

- Mário Santiago de Carvalho (Universidade de Coimbra): Francisco Suárez e a alteridade feminina.

- David González Ginocchio (Universidad Internacional de La Rioja): Voluntad y autodeterminación en Suárez.

- Leopoldo José Prieto López (Universidad Francisco de Vitoria): La génesis teológica de las Disputationes metaphysicae de Francisco Suárez.

A diferencia de los Congresos anteriores, se trata ahora de presentar lo que está previsto hacer. En este sentido tenemos noticias de tres Congresos.

\section{5. Ávila. «Congreso Internacional con motivo del IV centenario de la muerte de Francisco Suárez»}

El primero de ellos se celebró en Ávila, organizado por la Universidad Católica de Ávila con el título Congreso Internacional con motivo del IV centenario de la muerte de Francisco Suárez, los días 9-11 de noviembre. El organizador, Victoriano Martín, Director del Centro de Estudios Economía y Filosofía Política Luis de Molina (Universidad Católica Santa Teresa de Jesús de Ávila (UCAV) que tiene como fin fundamental la promoción del estudio y la investigación de los pensadores hispanoportugueses de los siglos XV, XVI y XVII), inició el encuentro en el que participaron, como ponentes, estudiosos españoles y portugueses de distintas universidades, como: Alberto Medina González, María Nieves San Emeterio Martín (Universidad Rey Juan Carlos), José Antonio Calvo Gómez (Universidad Católica de Avila), Ángel M. Fernández Álvarez (Universidad Complutense de Madrid), $\mathbf{M}^{\mathrm{a}}$ Idoya Zorroza Huarte (Universidad de Navarra), José Manuel Sánchez Caro (Universidad Pontificia de Salamanca y Universidad Católica de Ávila), José Carlos Martín de la Hoz (Academia de Historia Eclesiástica), Francisco Gómez Camacho (Universidad Pontificia Comillas), Juan Ramón Rallo (Instituto Juan de Mariana, Centro de estudios OMMA y IE University), Pedro Calafate (Universidade de Lisboa), Margarida Pires Seixas (Universidade de Lisboa), Francisco Tadeo Baciero Ruiz (Universidad de Salamanca), y Eduardo Fernández García. 


\subsection{Lisboa. Conferência Internacional: «Suárez em Lisboa 1617-2017»}

El segundo fue la Conferência Internacional: «Suárez em Lisboa 1617-2017» que tuvo lugar en la Faculdade de Direito de la Universidade de Lisboa los días 4-6 de diciembre de 2017. En esta ocasión quien promueve el acto académico convocado por los 400 años de la muerte de Francisco Suárez, es el Centro de Investigación: Teoria e História do Direito, Centro de Investigação da ULisboa (THD-ULisboa), con el apoyo de la Faculdade de Direito de la Universidade de Lisboa (FDL), del Instituto de História do Direito e do Pensamento Político (IHDPP) y de la Fundação para a Ciência e a Tecnologia (FCT).

El evento estuvo organizado por la Comisión Ejecutiva compuesta por Pedro Caridade de Freitas (Universidade de Lisboa), Margarida Seixas (Universidade de Lisboa) y Ana Caldeira Fouto (Universidade de Lisboa). Se trata de una Comisión muy bien acompañada por una excepcional Comisión Científica presidida por Martim de Albuquerque (Universidade de Lisboa) y constituida por los siguientes miembros: Alejandro Guzmán Brito (Pontificia Universidad Católica de Valparaíso), António Pedro Barbas Homem (Universidade de Lisboa), Eduardo Vera-Cruz Pinto (Universidade de Lisboa), Francisco Carpintero Benítez (Universidad de Cádiz), Georges Martyn (Universiteit Gent), José Artur Duarte Nogueira (Universidade de Lisboa), José Luis Fuertes Herreros (Universidad de Salamanca), José Meirinhos (Universidade do Porto), Juan Cruz Cruz (Universidad de Navarra), Leonel Ribeiro dos Santos (Universidade de Lisboa), Mário Santiago de Carvalho (Universidade de Coimbra), Pedro Calafate (Universidade de Lisboa), Randal Lessafer (Tilburg University / Katholieke Universiteit Leuven).

\subsection{Sevilla: International symposium on jesuit studies: «Francisco Suárez (1548-1617): Jesuits and Complexities of Modernity».}

Finalmente, el último evento del que tenemos noticia será el gran encuentro del año académico del Aniversario (curso 2017-2018), co-organizado por las instituciones de la Compañía de Jesús, con una gran diligencia, competencia académica, cuidado organizativo y de gestión; principalmente el Institute for Advanced Jesuit Studies at Boston College y el Departamento de Humanidades y Filosofía de la Universidad Loyola Andalucía, bajo la responsabilidad de los profesores Robert Aleksander Maryks (maryks@bc.edu) y Juan Antonio Senent de Frutos (jasenent@uloyola.es) respectivamente. El Congreso tendrá lugar los días 1-2 de junio de 2018 en Sevilla, con el título: "International symposium on jesuit studies: "Francisco Suárez (1548-1617): Jesuits and Complexities of Modernity», y en él se pretende estudiar «la obra de Suárez y de otros jesuitas de su época en el contexto de las diversas tradiciones que confluyeron en Europa entre el tardo medioevo y el Renacimiento y la primera modernidad». Se trata de plantearse cuestiones sobre la obra de la Compañía de Jesús como una modernidad alternativa y que se sustantiva en preguntas como: «¿Cuál es la relación entre la tradición ignaciana y jesuita y el desarrollo de la obra de 
Suárez y sus contemporáneos? ¿Qué elementos de la obra de Suárez y otros jesuitas pueden ser hoy relevantes para enfrentar la crisis de la modernidad? „ ${ }^{15}$.

El Congreso es, a su vez, un acto más del cuidado que la Universidad Loyola Andalucía está haciendo del pensamiento de Francisco Suárez en particular y del tesoro intelectual de la Compañía de Jesús en general. Pues, de hecho, la Universidad impulsa un proyecto de estudio y traducción de la obra del jesuita Francisco Suárez. Se trata de un trabajo realizado por un grupo de investigadores de los departamentos de Humanidades y Filosofía y de Derecho de la Universidad Loyola Andalucía, encabezado por el profesor Juan Antonio Senent. Concretamente este grupo ha iniciado un proyecto de investigación cuyo principal resultado será el estudio, traducción y edición en castellano e inglés de los cinco Tratados Morales del jesuita granadino. Un proyecto internacional que cuenta con la participación de un equipo de investigadores internacionales, entre ellos, de Marquette University, Cornell University, Sacred Heart de Nueva York, o de la Universidad de París.

\section{Publicaciones}

Dentro de esta presentación de actividades académicas e intelectuales en el IV Centenario de la muerte de Francisco Suárez hemos de señalar algunas publicaciones que durante el 2017 se han realizado o se están gestando en la medida que tenemos noticia. Sin ser exhaustivos, creo que dan noticia de lo que se está pensando. Muchas de las publicaciones nacen al albur de los Encuentros, Congresos y Jornadas que hemos señalado.

\subsection{Revistas científicas - Publicaciones periódicas}

Hoy en día la publicación en revistas de prestigio constituye uno de las visualizaciones y transmisión de conocimiento más valorado en el ámbito de la investigación. En este sentido la nómina de publicaciones que vamos a señalar es eco y reflejo de la calidad del pensamiento que genera la obra suareciana.

En primer lugar deberíamos señalar la obviedad y es el número en el que sale publicada esta breve crónica. El hecho de que Pensamiento. Revista de Investigación e Información Filosófica publique estudios sobre Suárez es de justicia al ser una publicación periódica editada por la Universidad Pontificia Comillas (Departamento de Filosofía y Humanidades I Facultad de Ciencias Humanas y Sociales). Y es una buena noticia para los estudios sobre Suárez pues esta revista es una publicación de alta calidad científica reconocida por bases de datos tan prestigiosas como Arts and Humanities Citation Index, Scopus, IBZ Online, Periodicals Index Online, MLA - Modern Language Association Database, Philosopher's Index, DOAJ y DIALNET

15 Disponible en https://www.bc.edu/centers/iajs/Programs/International-Symposia/ 2018-Seville.html. Consultado el 24 de setiembre de 2017. 


\section{A. Anales de la Cátedra Francisco Suárez.}

Anales de la Cátedra Francisco Suárez (ACFS), presente en bases de datos (Periodicals Index Online, Philosopher's Index, DOAJ, DIALNET, ERIHPlus) es una prestigiosa revista dentro del ámbito de filosofía jurídica y política editada por el Departamento de Filosofía del Derecho de la Universidad de Granada desde el año 1961. Evidentemente su nombre, su adscripción y su localización eran una invitación explícita a no pasar por delante ni olvidar la efemérides. De ahí que su volumen 51 (2017) haya tenido como título monográfico: Francisco Suárez en la vida de su tiempo y en la del nuestro ${ }^{16}$. Así lo ha expresado la directora de la revista en la editorial del volumen:

Con respecto al número 51 que ahora ve la luz, sobran las palabras. Desde que en 1948 la revista Pensamiento dedicara el Volumen IV a la figura de Francisco Suárez, con motivo de su nacimiento, ninguna otra ha vuelto a recuperarlo de manera tan global y profunda. Basta con ojearlo y hojearlo para apenas descubrir que estamos ante un monográfico-referente internacional en torno a la figura del insigne jurista Francisco Suárez y a su notable influencia pasada, presente y, mucho me temo que, futura en el panorama jurídico y político. Espero que disfruten tanto de su lectura como yo de su edición ( $p .3)$.

Presentamos el elenco de los estudios, que lógicamente por el carácter jurídico de la revista dedicada a la filosofía jurídica o del derecho, se circunscriben a estudios sobre el pensamiento filosófico-jurídico del maestro granadino:

- Juana María Gil Ruiz (Universidad de Granada), «Francisco Suárez en la vida de su tiempo y en la del nuestro», pp. 5-6.

- Antonio Enrique Pérez Luño (Universidad de Sevilla), «Francisco Suárez y la Filosofía del Derecho actual (Aspectos de su pensamiento jurídico ante el Cuarto Centenario de su muerte)», pp. 9-25.

- Rosa Colmenarejo Fernández (Universidad Loyola Andalucía), «El problema de la felicidad en Aristóteles: respuestas desde Francisco Suárez y Martha Nussbaum», pp. 27-47.

- Franco Todescan (Università degli Studi di Padova), «Francisco Suárez y la razón de Estado en el contexto de la literatura española del siglo XVII», pp. 49-65.

- Pablo Badillo O’farrell (Universidad de Sevilla), «Revisita a Suárez y Vico», pp. 67-77.

- Milagros Otero Parga (Universidad de Santiago de Compostela), «¿Influyó Suárez en el pensamiento de Andrés Bello?», pp. 79-100.

- Manuel Salguero Salguero (Universidad de Granada), «Proporcionalidad y elaboración suareciana de la atribución intrínseca como fundamento filosófico de la analogia iuris y de la analogia legis», pp. 101-128.

16 Disponible en http://revistaseug.ugr.es/index.php/acfs/issue/view/482/showToc, consultado el 21 de septiembre de 2017. 
- Alba Jiménez Rodríguez (Universidad Autónoma de Madrid), «El concepto de especie intencional en Suárez: su proyección en la Filosofía de la Escuela Alemana y en algunas corrientes de la Filosofía contemporánea», pp. 129-142.

- Juan Carlos Utrera García (Universidad Nacional de Educación a Distancia - UNED), «El fundamento de la validez jurídica en Francisco Suárez. Claves para una lectura contemporánea», pp. 143-162.

- Adolfo Sánchez Hidalgo (Universidad de Córdoba), «Vitoria y Suárez: el Derecho Internacional en el tránsito a la modernidad», pp. 163-182.

- Pablo Font Oporto (Universidad Loyola Andalucía), «Tipos de tirano y resistencia en Francisco Suárez», pp. 183-207.

- Luis Carlos Amezúa Amezúa (Universidad de Valladolid), «La potestad tributaria en Francisco Suárez», pp. 209-231.

- Lorenzo Milazzo (Universidad de Pisa), «Suárez: La verdad de la Ley y su Autoridad», pp. 233-255.

- Manuel Lázaro Pulido (CEDEU - Universidad Rey Juan Carlos / Universidad Bernardo O’Higgins), «Fundamentos teológicos de la censura en Francisco Suárez», pp. 257-275.

- Giannina Burlando (Pontificia Universidad Católica de Chile), «Psicología moral en Suárez y Descartes: La pasión en la vida virtuosa», 277-302.

\section{B. Anuario filosófico}

En la tónica de lo que venimos viendo, la revista Anuario filosófico también se ha sumado a la fiesta intelectual a la que la comunidad intelectual ha sido convocada en torno a Francisco Suárez, dedicándole consecuentemente un número monográfico a su pensamiento y su obra. La revista es una publicación del Departamento de Filosofía de la Universidad de Navarra de gran prestigio (bases de datos: Arts and Humanities Citation Index, Scopus, Academic Search Premier, Fuente Academica Plus, IBZ Online, Periodicals Index Online, L'Année philologique, Linguistics \& Language Behavior Abstracts, Philosopher's Index, vLex, DIALNET) que se ha sumado.

El volumen monográfico, como reconocen los propios editores, nace con la vocación de aportar a la celebración del cuarto centenario del fallecimiento de Francisco Suárez (1548-1617), una reactualización de su pensamiento de este jesuita español en su carácter desde una mirada filosófica y multidisciplinar: desde la metafísica, la psicología filosófica, la filosofía política, el derecho y la economía a la teoría de la acción. Con ello, como señala la editorial, se busca abordar desde el panorama contemporáneo de estas disciplinas las tesis de este autor complejo y controvertido, puente y gozne entre el pensamiento medieval y el moderno.

Se trata del volumen 50, no 2 de 2017, bajo el epígrafe: Francisco Suárez (1548-1617): Escolástica y mundo moderno. El número presenta cinco trabajos con los que mostrar la riqueza de planteamientos del filósofo y teólogo jesuita: 
- Víctor Sanz Santacruz (Universidad de Navarra), M ${ }^{a}$ Idoya Zorroza (Universidad de Navarra), «Francisco Suárez (1548-1617): Escolástica y mundo moderno", pp. 259-266.

- Fernando Hernández Fradejas (Universidad de Valladolid), «Derecho de propiedad privada y fiscalidad en Francisco Suárez», pp. 269-296.

- Miguel Saralegui (Universidad Adolfo Ibañez), La conciliación de lo político y lo religioso. Suárez y Hobbes sobre la potestad indirecta», pp. 297-321.

- Daniel Heider (University of South Bohemia), «Suárez on sound and hearing», pp. 323-344.

- Mauricio Lecón (Universidad Panamericana), «Recuperación suareciana de

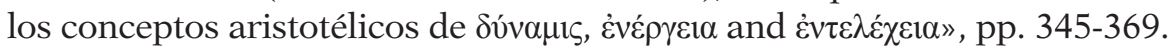

- David González Ginocchio (Universidad Internacional de La Rioja), «Praxis and the ends of action in Suárez», pp. 371-396.

\section{Cauriensia}

Cauriensia. Revista anual de Ciencias Eclesiásticas, es una revista editada por el Instituto Teológico de Cáceres (Centro afiliado a la Facultad de Teología de la Universidad Pontificia de Salamanca) y coeditado con la Universidad de Extremadura y la Editorial Sindéresis. Tanto el Instituto Teológico de Cáceres, como la Editorial Sindéresis han estado muy presentes en la organización y participación de este año suareciano. La revista que está presente en importantes bases de datos (Emerging Sources Citation Index, Scopus, Fuente Academica Plus, ATLA Religion Database, DOAJ, DIALNET, ERIHPlus).

En su volumen XII (2017) dedicará su sección como Monográfico a recordar la figura de Suárez, bajo el título Francisco Suárez entre Escolástica y Mundo Moderno. Editado por los organizadores de las citadas Reuniones Filosóficas de la Universidad de Navarra (cf. el epígrafe 2.3), que están actualmente siendo seleccionados y evaluados, se editarán algunos trabajos escritos por Cintia Faraco (Università degli Studi de Napoli «Federico II»), Ilaria Acquaviva. (Scuola Alti Studi Collegio San Carlo di Modena), Szilárd Tattay (Institute for Legal Studies, Hungarian Academy of Sciences), Daniel Heider (University of South Bohemia), Ivone Moreira (Universidade Católica Portuguesa), José Ángel García Cuadrado (Universidad de Navarra), Agustín Echavarría (Universidad de Navarra), Costantino Esposito (Università degli Studi de Bari Aldo Moro), Álvaro Perpere (Universidad Católica Argentina), Jean-Paul Coujou (Institute Catholique de Toulouse/Institut Michel Villey), Leopoldo José Prieto López (Universidad Francisco de Vitoria), Jesús Villagrasa Lasaga (Ateneo Pontificio Regina Apostolorum) y Sameer Advani (Ateneo Pontificio Regina Apostolorum).

\section{Comprendre}

Aunque de manera transversal, la revista Comprendre, si bien no va a dedicar un número a la memoria exclusiva de Suárez, sí que en honor a su figura ha 
pensado elaborar para el 2018 un número dedicado al barroco español, en el que se incluirán estudios sobre le pensamiento de Suárez y del contexto barroco peninsular. Comprendre. Revista catalana de filosofía de la Facultat de Filosofia de la Universitat Ramon Llull y editada por la Editorial Herder está presente en diversas bases de datos (Scopus, IBZ Online, Philosopher's Index, DIALNET, ERIHPlus).

Dirigido el número monográfico por Manuel Lázaro Pulido (Universidad Nacional de Educación a Distancia) y $\mathrm{M}^{\mathrm{a}}$ Idoya Zorroza Huarte (Universidad Pontificia de Salamanca), el número contará con tres estudios sobre Francisco Suárez:

- Vicente Llamas Roig (Instituto Teológico de Murcia OFM. Facultad de Teología Fundamental, Pontificia Università Antonianum), «La analogia entis en la metafísica suarista. Notas para un patrón mixto».

- Leopoldo José Prieto López (Universidad Francisco de Vitoria), «Una clave de lectura de las Disputationes Metaphysicae: el actualismo de Suárez».

- Aleksandr Pogoniailo (St. Petersburg State University), «Del sujeto al sujeto (Terminología suareciana en la obra de Descartes)».

El volumen irá acompañado de otros estudios sobre el Barroco peninsular por parte de Patrícia Calvário (Universidade do Porto) con el estudio ««Per essentiam Deum videre» como limitação da omnipotência divina: A crítica de Gabriel Vásquez às concepções bizantinas acerca da visão de Deus»; Rafael Ramis Barceló (Universitat de les Illes Balears - IEHM), «Arnau de Vilanova i el pensament reformat» y en el que participarán también Juan Antonio Gomez Garcia (Universidad Nacional de Educación a Distancia), Manuel Lázaro Pulido (Universidad Nacional de Educación a Distancia) y $\mathbf{M}^{\mathrm{a}}$ Idoya Zorroza Huarte (Universidad Pontificia de Salamanca).

\section{E. Otros artículos}

Conscientes de que la presentación que estamos realizando tiene más un carácter testimonial que registral, sin embargo, nos parece oportuno citar al menos algunos artículos publicados en revistas peninsulares o por autores peninsulares y que señalamos a continuación, sabiendo que esta onomástica ha propiciado una renovación de los estudios suarecianos a nivel internacional de cuya difusión e impacto tendremos noticia sólo con posterioridad:

- Pedro Calafate, «A idea de Comunidade universal em Francisco Suárez», IHS: Antiguos Jesuitas en Iberoamérica, 5/20 (2017), pp. 48-65.

- Pablo Font Oporto, «Obediencia y desobediencia a reyes herejes y no cristianos en Francisco Suárez. Potestad del papa al respecto», Gregorianum,. 98 (2017), pp. 61-74.

- Josep Giménez Melià, «Francisco Suárez: intérprete y defensor de la espiritualidad ignaciana», Manresa. Revista de Espiritualidad Ignaciana, 89/351 (2017), pp. 169-178. 
- José Ignacio Herce Maza, «La interpretación de las normas: Francisco Suárez y los límites del silogismo», Gabilex. Revista del Gabinete Jurídico de Castilla-La Mancha, 10 (2017), pp. 78-153.

- Antonio Enrique Pérez Luño, «De la norma al ordenamiento jurídico: aspectos de la doctrina jurídica de Francisco Suárez», Revista de fomento social, 285 (2017), pp. 73-84.

- Leopoldo José Prieto López, «Actualidad de Francisco Suárez, en el cuarto centenario de su óbito (1617-2017)», Comunicación y hombre, 13 (2017).

- Rodrigo Tena, «Francisco Suárez, jurista», El notario del siglo XXI. Revista del Colegio Notarial de Madrid, 68 (2016), pp. 202-205.

\subsection{Libros}

Siguiendo la estela de los Congresos, Encuentros... realizados se siguen publicaciones colectivas. Algunos de ellos, como hemos visto, se han realizado o realizarán bajo el formato de número monográfico de una revista, un formato exigido —ya hemos señalado— por las agencias de calidad y acreditación; pero otros inspirarán la edición de algunos números colectivos. Evidentemente, estas obras están en proceso de edición, no obstante podemos aventurar y presentar algunos de los libros que serán publicados.

A partir de los resultados de los trabajos escritos en Salamanca se publicará un volumen editado por José Luis Fuertes Herreros, Manuel Lázaro Pulido, Ángel Poncela y $\mathrm{M}^{\mathrm{a}}$ Idoya Zorroza, titulado Francisco Suárez: Entre el Renacimiento y la Modernidad, que será publicado en Eunsa o en la Editorial Sindéresis.

Teniendo en cuenta los materiales del Encuentro celebrado en Porto-Coimbra, con un título a determinar pero que girará en torno a la temática del Congreso (Os fundamentos antropológicos e teóricos do pensamento jurídico e político em $F$. Suárez e na Segunda Escolástica: contexto e projecções), se está preparando una coedición entre el IEF - Instituto de Estudos Filosóficos. Faculdade de Letras da Universidade de Coimbra, dentro de la Coleção e-ovodLibet y la Editorial Sindéresis en su Colección Biblioteca de Humanidades Salmanticensis, Serie Filosofia. La publicación contará con un doble formato: digital en repositorio abierto y en papel, contará con dos ISBN.

Por su parte el International symposium on jesuit studies: «Francisco Suárez (1548-1617): Jesuits and Complexities of Modernity», prevé publicar una selección de estudios presentados en el Congreso en un volumen a determinar. Tienen previsto que sea bien en Jesuit Studies Series de Brill o en el Journal of Jesuit Studies.

$$
* * *
$$

Podríamos concluir que los actos realizados en la Península Ibérica corresponden a la figura histórica y la proyección del pensamiento y obra de Francisco Suárez y señalan su interés desde el punto de vista de la historia del pensamiento (filosófico, teológico y jurídico) como la potencialidad de su 
propuesta para un siglo XXI necesitado de una apuesta por la reflexión teóricopráctica fundamentada un unas bases que siendo sólidas siempre están abiertas a la relectura y reactualización.

Los jesuitas siempre han estado impelidos, desde su fundación, a llegar a los confines intelectuales, a las nuevas fronteras (geográficas y mentales) ${ }^{17}$, desde una metodología propia, no exenta de visiones y discusiones internas ${ }^{18}$, que pudiera poner un sólido fundamento a una nueva teología capaz de realizar una verdadera aportación al pensamiento de la época y a las mentes de su tiempo. Esto suponía profundizar la teología escolástica dirigida a la propia conciencia como espacio de la práctica, una teología humanista: práctica, científica, cultural, pedagógica ${ }^{19}$. Ello supuso la adopción de una nueva metodología, en la que la visión sistemática de corte especulativo se relacionara con la visión pragmático-moral, una metodología que tuvo como protagonistas de lo primero a Francisco Suárez y de lo segundo a Luis de Molina (1535-1600) y que tiene en la ciencia de la reflexión jurídica el elemento que aúna teoría y práctica sobre el hombre en su complejidad comunitaria (social y eclesial). De este modo Francisco Suárez diseñó un pensamiento abierto a la penetración en la cultura a la que ofrece su pensamiento a los lecturas de su obra en todo momento, más aún ahora en los límites intelectuales del siglo XXI.

UNED / U. Bernardo O’Higgins

mlazarop@fsof.uned.es

Universidad Pontificia de Salamanca

izorrozahu@upsa.es
Manuel Lázaro Pulido

$M^{\mathrm{a}}$ IDOYa ZorRoZA

[Artículo aprobado para publicación en enero de 2018]

17 Pino-Díaz, F. del, «Los métodos misionales de los jesuitas y la cultura de «los otros»», en Hernández, J. J. y Moreno, R (eds.), La misión y los jesuitas en la América española, 15661767, cambios y permanencias, Sevilla, CSIC, 2005, pp. 43-68.

18 FÉdou, M., Les théologiens jésuites: Un courant uniforme?, Brussels, Lessius, 2014.

19 Cf. Giampietro, G., Trossarelli, F., «La pedagogía nella tradizione culturale dei gesuiti», en Nuove questioni di storia della pedagogía. Vol. I, Brescia, La Scuola, 1977, pp. 737-770. 